C-A/AP/\#143

March 2004

Hadron Beam Emittance Growth Due to Electron Beam Parameter Jitter in Linac-Ring Electron-Ion Colliders

\author{
C. Montag, BNL
}

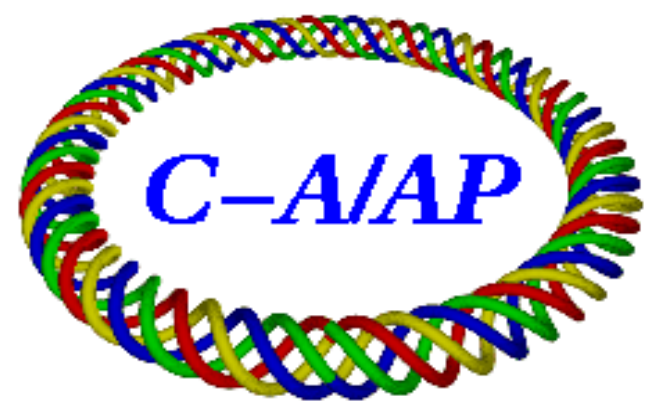

Collider-Accelerator Department

Brookhaven National Laboratory

Upton, NY 11973 


\title{
Hadron Beam Emittance Growth Due to Electron Beam Parameter Jitter in Linac-Ring Electron-Ion Colliders
}

\author{
C. Montag
}

March 11, 2004

\begin{abstract}
We study the effect of bunch-to-bunch variations of the electron bunch intensity and rms transverse beam size on the hadron beam emittance in linac-ring electron-ion colliders, using a linearized model of the beambeam force. Upper limits for the rms jitter amplitudes of these quantities are given for various linac-ring electron-ion colliders.
\end{abstract}

\section{Introduction}

In recent years, several linac-ring electron-ion colliders have been proposed, namely eRHIC at BNL [1], ELIC at Jefferson Lab [2], THERA at DESY [3], and the QCD Explorer QCDE at CERN [4]. Table 1 lists some design parameters of these facilities.

A destinctive feature of all linac-ring colliders is the fact that each electron bunch collides with the ion beam only once before it is sent to the beam dump. While this overcomes a beam-beam tuneshift limitation on the electron beam of ring-ring colliders, it also introduces noise into the ion beam, via the beam-beam interaction with electron bunches of fluctuating intensity and transverse size. To study this effect and compute upper limits on bunch-to-bunch intensity and size variations, we assume that these fluctuations do not exhibit any bunch-to-bunch

\begin{tabular}{|ll|c|c|c|c|}
\hline & & eRHIC & ELIC & THERA & QCDE \\
\hline beam-beam tuneshift & $\xi_{x, y}$ & 0.007 & 0.006 & 0.0025 & 0.004 \\
collision frequency & $f_{\mathrm{c}}[\mathrm{kHz}]$ & 78 & 250 & 0.28 & 0.1 \\
\hline tolerable jitter & $\sigma_{\xi} / \xi$ & $9.5 \cdot 10^{-4}$ & $6.3 \cdot 10^{-4}$ & 0.045 & 0.047 \\
\hline
\end{tabular}

Table 1: Parameters of the linac-ring electron-ion colliders eRHIC, ELIC, THERA, and QCDE. The collision frequency $f_{c}$ is defined as the number of collisions per second for one particular ion bunch. 
correlations, i.e. that they resemble white noise. Furthermore, we linearize the beam-beam force to enable us to derive analytical expressions for the jitter tolerances.

\section{Theory}

The beam-beam kick for round beams can be expressed as

$$
\begin{aligned}
\Delta z^{\prime} & =-2 \frac{r_{p}}{\gamma} \frac{N_{e}}{r^{2}} z\left(1-\exp \left(-r^{2} / 2 \sigma^{2}\right)\right), \\
r^{2} & =x^{2}+y^{2}, z=x, y
\end{aligned}
$$

where $r_{p}=1.54 \cdot 10^{-18} \mathrm{~m}, \gamma, N_{e}$, and $\sigma$ denote the classical proton radius, the Lorentz factor of the hadron beam, the number of electrons per bunch, and the electron rms transverse beam size, respectively. This expression can be linearized for $z \ll \sigma$ as

$$
\begin{aligned}
\Delta z^{\prime} & =-2 \frac{r_{p}}{\gamma} \frac{N_{e}}{r^{2}} z\left(1-\sum \frac{\left(-r^{2} / 2 \sigma^{2}\right)^{n}}{n !}\right) \\
& \approx-2 \frac{r_{p}}{\gamma} \frac{N_{e}}{r^{2}} z \frac{-r^{2}}{2 \sigma^{2}} \\
& =\frac{r_{p}}{\gamma} \frac{N_{e}}{\sigma^{2}} z \\
& =-\frac{4 \pi \xi}{\beta^{*}} \cdot z \\
& =k \cdot z
\end{aligned}
$$

which resembles a quadrupole kick. Introducing normalized coordinates $z_{N}$ and $z_{N}^{\prime}$, this finally becomes

$$
\Delta z_{N}^{\prime} \approx-4 \pi \xi z_{N} .
$$

At the interaction point (IP), where it is assumed that $\alpha=0$, the action $J$ before the quadrupole kick can be expressed as

$$
J_{i}=z_{N}^{2}+z_{N}^{\prime 2} .
$$

After experiencing the beam-beam kick with its fluctuating strength $\delta \xi$, the action is

$$
\begin{aligned}
J_{f} & =z_{N}^{2}+\left(z_{N}^{\prime}-4 \pi \delta \xi z_{N}\right)^{2} \\
& =z_{N}^{2}+z_{N}^{\prime 2}-8 \pi \delta \xi z_{N} z_{N}^{\prime}+(4 \pi)^{2}(\delta \xi)^{2} z_{N}^{2} .
\end{aligned}
$$

The change in action due to the kick is therefore

$$
\begin{aligned}
\Delta J & =J_{f}-J_{i} \\
& =-8 \pi \delta \xi z_{N} z_{N}^{\prime}+(4 \pi)^{2}(\delta \xi)^{2} z_{N}^{2} .
\end{aligned}
$$


Averaging $\Delta J$ over many turns, the first term vanishes, and we get with $\left\langle z_{N}^{2}\right\rangle=J / 2$

$$
\begin{aligned}
\frac{\langle\Delta J\rangle}{J} & =8 \pi^{2}\left\langle(\delta \xi)^{2}\right. \\
& =8 \pi^{2} \xi^{2} \frac{\left\langle(\delta \xi)^{2}\right\rangle}{\xi^{2}} .
\end{aligned}
$$

\section{$3 \quad$ Numerical examples}

The action (or emittance) doubling rate $\tau_{2}^{-1}$ can be expressed as

$$
\begin{aligned}
\tau_{2}^{-1} & =\frac{1}{J} \frac{\mathrm{d} J}{\mathrm{~d} t} \\
& =f_{c} \cdot \frac{\langle\Delta J\rangle}{J} \\
& =f_{c} \cdot 8 \pi^{2} \xi^{2} \frac{\left\langle(\delta \xi)^{2}\right\rangle}{\xi^{2}} .
\end{aligned}
$$

To limit the luminosity degradation due to this emittance growth to tolerable numbers, the emittance doubling time should be larger than the minimum acceptable time $T_{2}$,

$$
\tau_{2}>T_{2}
$$

This requirement yields an expression for the maximum relative rms jitter of the beam-beam parameter

$$
\frac{\left\langle(\delta \xi)^{2}\right\rangle}{\xi^{2}}<\frac{1}{T_{2} f_{c} \cdot 8 \pi^{2} \xi^{2}}
$$

Results for the various linac-ring colliders are listed in Table 1, assuming a tolerable emittance doubling time of $3600 \mathrm{sec}$.

\section{Simulation results}

While the effect of fluctuations in the linearized case can be calculated analytically, study of the realistic nonlinear situation requires numerical simulations. To compare the simulation code with the analytical calculations, the linear case was simulated using eRHIC parameters (see Table 1) and a three percent relative rms jitter of the beam-beam parameter $\xi$. The evolution of the normalized particle action $J \cdot \beta / \sigma^{2}$ for this case shows perfect agreement between analytical results and simulations, see Figure 1.

In the nonlinear case, a three percent relative jitter fo the beam-beam parameter $\xi$ can occur due to $3 \%$ fluctuations of the electron bunch population, $N_{e}$, or $1.5 \%$ fluctuations in the electron beam size, $\sigma_{z}$. While in the linear case these fluctuations are equivalent, they are expected to act differently in the nonlinear 


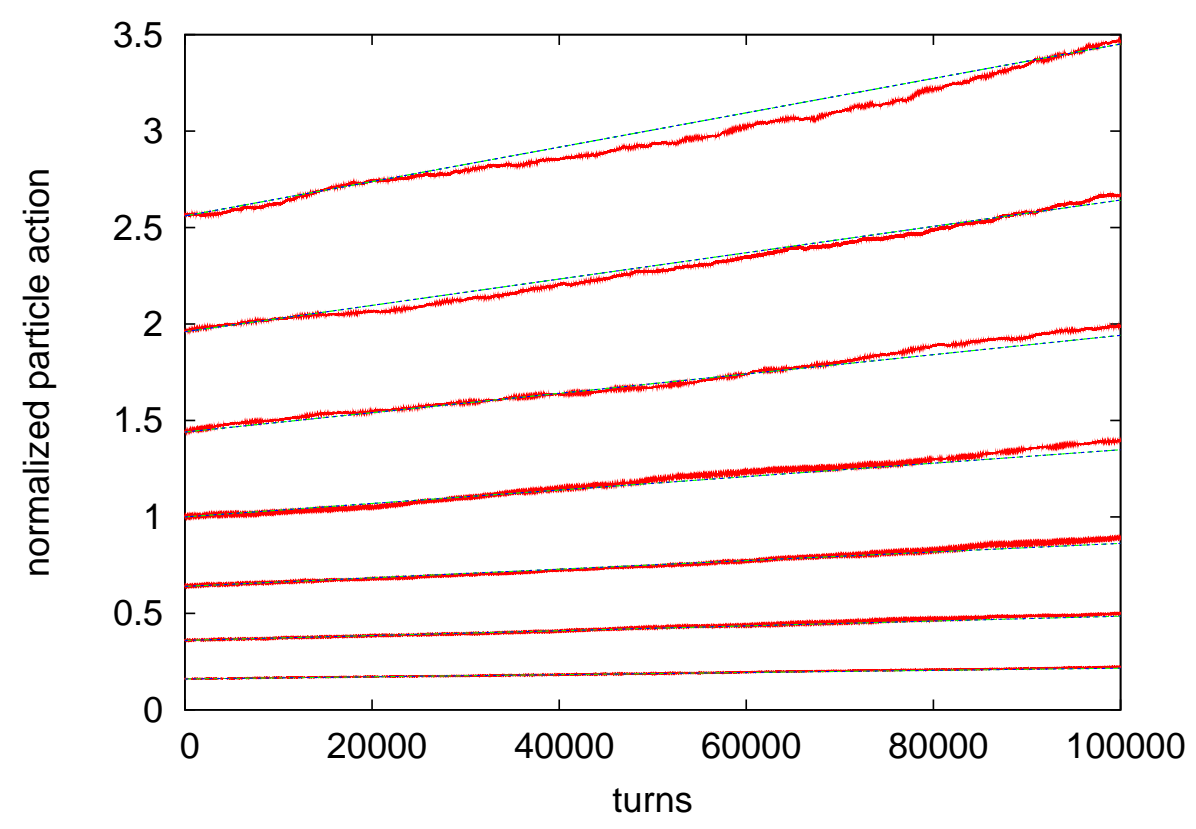

Figure 1: Normalized particle action $J \cdot \beta / \sigma^{2}$ vs. turn number for different initial values of $J$, with the beam-beam force linearized according to Equation 3. The straight, blue lines correspond to the analytical result, Equation 9, while the red lines show simulated data.

case, Equation 1.

Figure 2 shows the growth of the normalized particle action for a three percent relative rms jitter of the electron bunch intensity $N_{e}$. While for small amplitudes below about one $\sigma$ the agreement with the result of the linearized calculation is very good, the linearized model overestimates the growth for amplitudes greater than one $\sigma$.

For a relative rms jitter of three percent of the inverse squared electron beam size $\sigma_{z}^{-2}$, the overestimation by linearization becomes even more apparent, while below one $\sigma$ the agreement with the linear model is again very good, see Figure 3 .

\section{Conclusion}

An analytic expression for the maximum tolerable rms jitter of the beam-beam parameter due to bunch-to-bunch fluctuations of the electron beam parameters in electron-ion colliders has been derived, using a linearized approximation of the beam-beam force. Simulations show that this approximation is justified for small particle amplitudes, while it significantly overestimates the resulting 


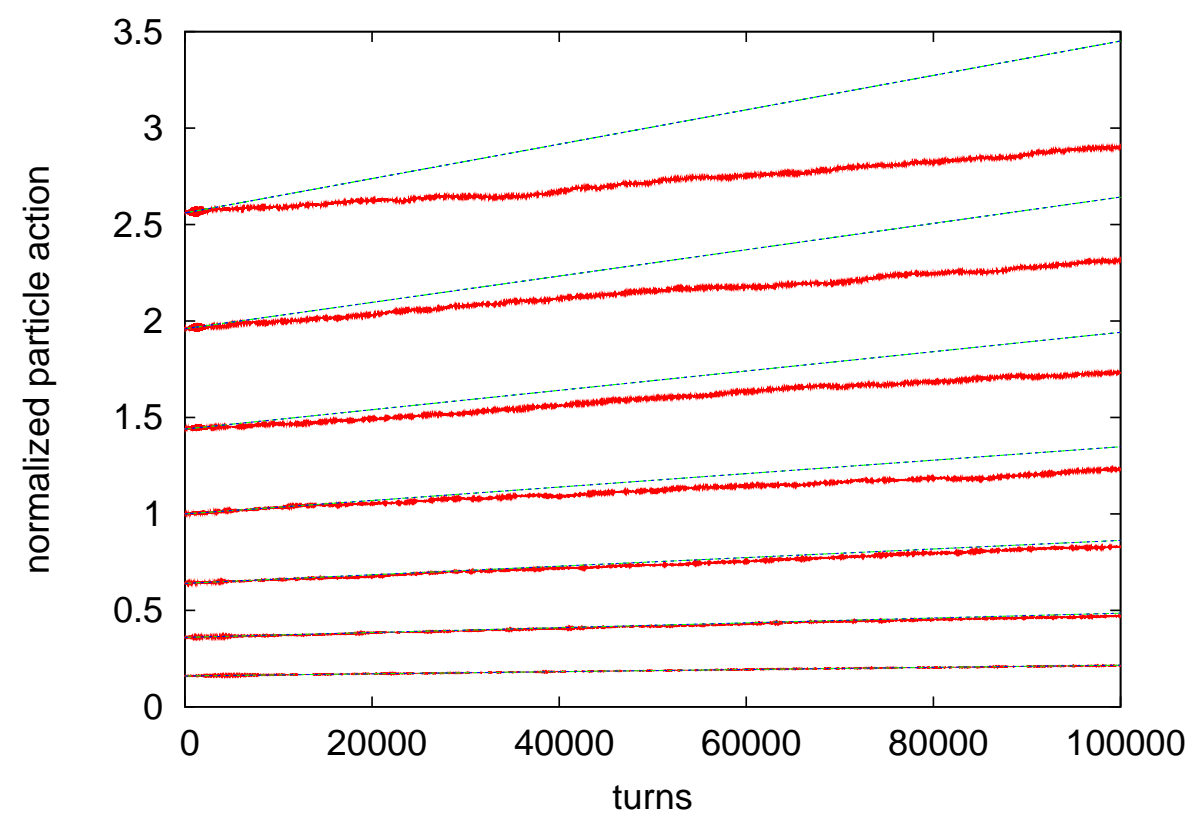

Figure 2: Normalized particle action $J \cdot \beta / \sigma^{2}$ vs. turn number for different initial values of $J$. The analytical result for the linearized beam-beam force is indicated by blue lines, while the red lines show simulation data for the nonlinear beambeam force with fluctuating electron beam intensity $N_{e}$. The rms width of the fluctuation is set to $\sqrt{\left\langle\left(N_{e}-\left\langle N_{e}\right\rangle\right)^{2}\right\rangle} /\left\langle N_{e}\right\rangle=0.03$.

growth of particle action for particles beyond one $\sigma$. However, this depletion of the beam core determines the associated luminosity degradation.

The calculated stability requirements for eRHIC and ELIC are quite tight, with $\sigma_{\xi} / \xi<0.001$, while the corresponding numbers for THERA and QCDE are significantly more relaxed, $\sigma_{\xi} / \xi<0.05$.

\section{Acknowledgments}

I would like to thank Steve Peggs for helpful, stimulating discussions and suggestions.

\section{References}

[1] eRHIC ZDR, C-A/AP/142

[2] L. Merminga, Y. Derbenev, ELIC: An Electron-Light Ion Collider at CEBAF, in: ICFA Beam Dynamics Newsletter No. 30, April 2003 


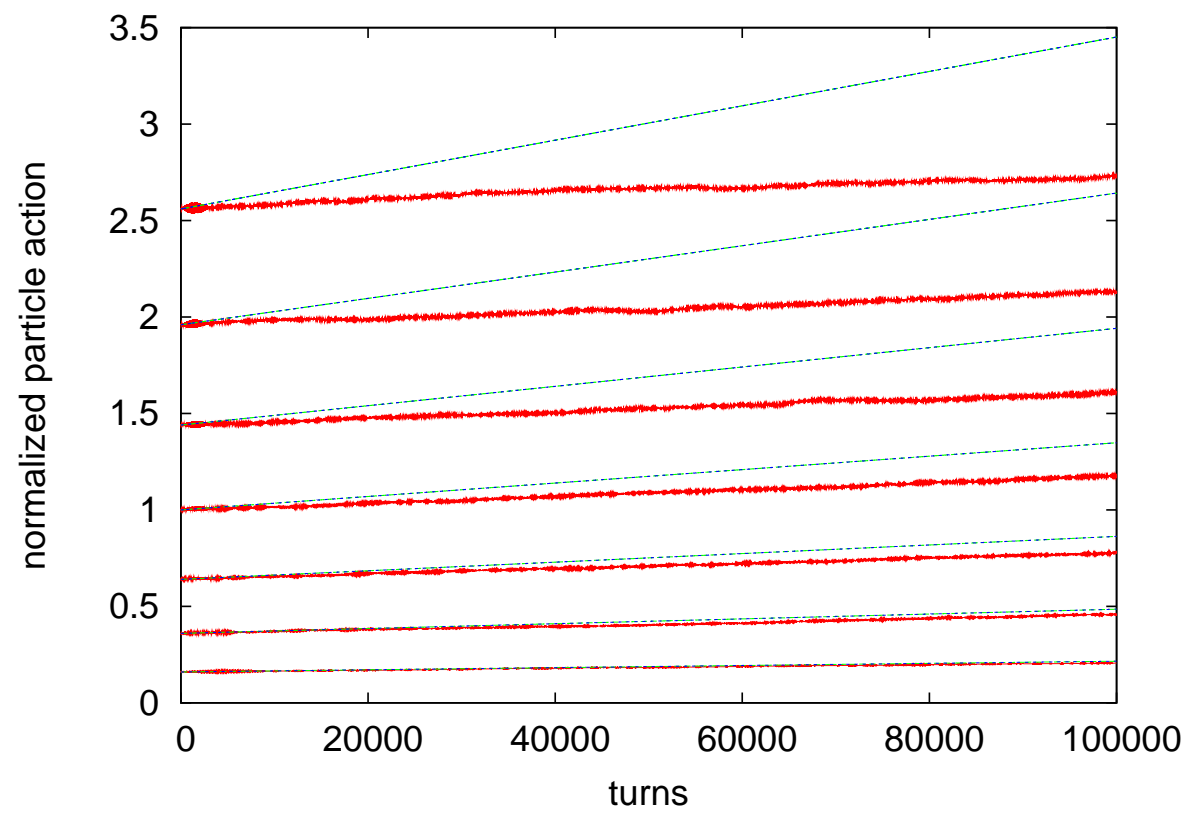

Figure 3: Normalized particle action $J \cdot \beta / \sigma^{2}$ vs. turn number for different initial values of $J$. The red lines show simulated data for the nonlinear beam-beam force with fluctuating electron beam size $\sigma_{z}$. The magnitude of the fluctuation is set to $\sqrt{\left\langle\left(\sigma_{z}^{2}-\left\langle\sigma_{z}^{2}\right\rangle\right)^{2}\right\rangle} /\left\langle\sigma_{z}^{2}\right\rangle=0.03$. The analytical result for the linearized beam-beam force is indicated by straight blue lines.

[3] THERA: Electron-Proton Scattering at a cms energy of $\approx$ $1 \mathrm{TeV}$, TESLA Technical Design Report, Part VI, Chapter 2, http://tesla.desy.de/new_pages/TDR_CD/PartVI/append.html

[4] D. Schulte, F. Zimmermann, QCD Explorer Based on LHC and CLIC, LHC Project Note 33, CLIC Note 589 